\title{
STRONG COUPLING OF OPTICAL MODES IN PHOTONIC MOLECULES WITH CDTE NANOCRYSTALS
}

\author{
Konstantin I. Rusakou ${ }^{1}$, Anatoly A. Gladyshchuk', Sergey V. Chugunov ${ }^{1}$, \\ Yury P. Rakovich ${ }^{1,2}$, John F. Donegan ${ }^{2}$, Andrey L. Rogach ${ }^{3}$, Nikolay Gaponik ${ }^{4}$ \\ 'Department of Physics, Brest State Technical University (BSTU), \\ 224017, BSTU, Brest, Belarus \\ Phone: 375-162-422643,Fax: 375-162-422127,e-mail: rusakov@bstu.by \\ ${ }^{2}$ Departments of Physics and Chemistry, Trinity College, Dublin 2, Ireland \\ Phone: 353-1-608-2193, Fax: 353-1-671-1759, e-mail: rakovicy@tcd.ie \\ ${ }^{3}$ Photonics \& Optoelectronics Group, Physic Department and CeNS, University of Munich, \\ 80799 Munich, Germany \\ Phone: 49-89-2180-1418, Fax: -3441,e-mail: Andrey.Rogach@physik.uni-muenchen.de \\ ${ }^{4}$ Institute of Physical Chemistry, University of Hamburg, 20146 Hamburg, Germany \\ Phone: 49-40-42838-3452, Fax: -7069, e-mail: gaponik@chemie.uni-hamburg.de
}

\begin{abstract}
We present a detailed study of the localized coupled-cavity modes in a photonic molecule formed from two dielectric spherical microcavities with $\mathrm{CdTe}$ nanocrystals. A layer-by-layer deposition technique provides controllable coating of the microspheres with a shell of close-packed nanocrystals of approximately 4 $\mathrm{nm}$ in diameter. The observed spectral structures originate from the coupling of the electronic transitions in nanocrystals and the photon states of interacting microspheres and, in analogy to the formation of molecular electronic orbits, can be assigned to bonding and antibonding photon localized states.
\end{abstract}

Keywords: coupled microcavities, semiconductor nanocrystals, whispering gallery modes, mode degeneracy

\section{INTRODUCTION}

In recent years the modification of photon modes in spherical microcavities has been of great interest both for studies of fundamental optical properties and for the potential applications [1]. It was shown that the luminescence from semiconductor nanocrystals (NC) such as CdSe or CdTe, can be enhanced or inhibited when these nano-emitters are placed on the surface of spherical microcavity, which supports emission propagation in discrete optical modes (whispering gallery modes (WGM)) [2,3]. Following the analogy with quantum mechanics, three integers, $n, l$ and $m$, describing WGM, correspond to angular, radial and the azimuthal quantum numbers, respectively. It was recently recognized that the partial delocalization of Mie resonance states in microspheres is of great importance because it suggests a possibility for coherent coupling between WGM of two adjacent spherical particles forming "photonic molecule (PM)" [4]. Although experimentally, the coupling of the photon modes in the PM can cause a narrow WGM resonance to split into two modes of lower quality factor [5], recent theoretical considerations [6] show more complex internal distribution of density of photonic states of PM. In this paper we report on the optical switching and observation of fine structure in PL spectra of PM, which can be interpreted as a result of lifting of the WGM degeneracy with respect to $m$.

\section{EXPERIMENTAL DETAILS}

Aqueous dispersions of MF microspheres, of $3 \mu \mathrm{m}$ in diameter, were combined with luminescent CdTe NCs using a layer-by-layer deposition technique. The colloidal solution of CdTe NCs, with a PL maximum at $563 \mathrm{~nm}$ (2.9 nm radius) and a PL quantum efficiency of $15 \%$ at room temperature, was used for coating MF microspheres. The PL spectra from 
microspheres were recorded using a RENISHAW micro-Raman system equipped with a positioning stage. $\mathrm{An}_{\mathrm{Ar}}{ }^{+}$laser $(\lambda=488 \mathrm{~nm}, 1.0 \mathrm{~mW}$ power) was used to provide excitation.

\section{RESULTS AND DISCUSSION}

To investigate the photon modes in interacting spherical microcavities we have measured PL spectra scanning a sample along the longitudinal axis of the PM (Fig.1).

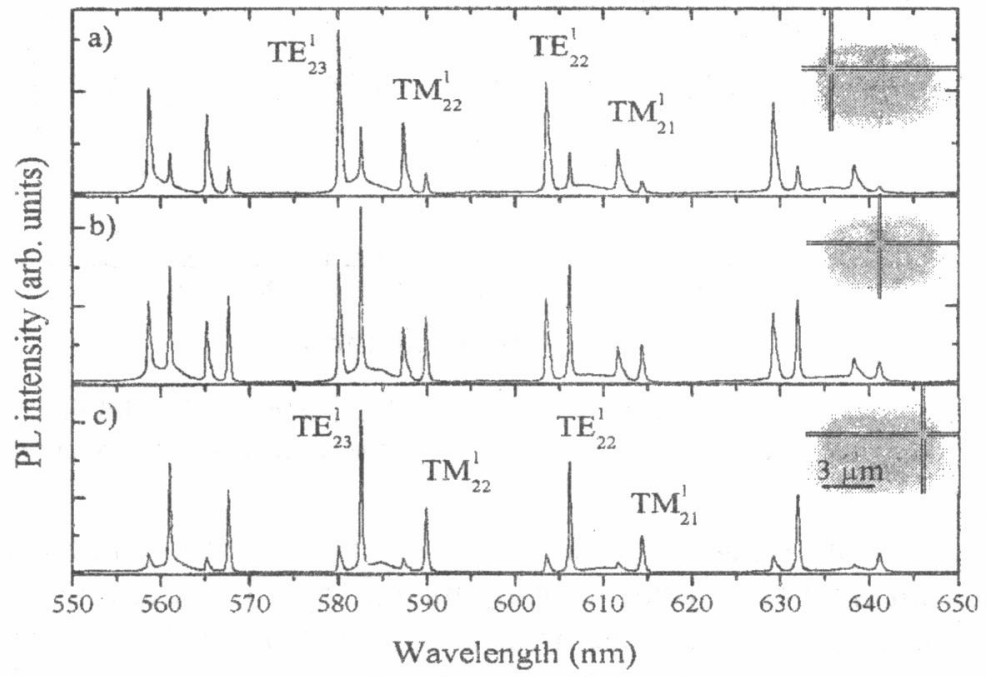

Fig. 1. - PL spectra of a PM with excitation and detection at three different positions along its longitudinal axis. Insets: microscope images of the PM, with the cross-hairs indicating the excitation-detection position.

The pronounced double structure, with the intensity distribution dependent on the excitation position (Fig. 1), is just a result of superposition of the uncoupled WGMs of individual microspheres. The lack of intermode coupling in this configuration is not surprising because the coupling between electro-magnetic fields of the spheres is expected to be maximum in the direction parallel to the PM axis, when some of WGM orbitals lie in the same plane [6]. However, the observed redistribution of intensity between the components of WGM double structure clearly demonstrates the propagation of the light along the PM and possibility of wavelength switching in the PM.

As predicted in Ref. [6], controllable alignment geometry of the PM is crucial in order to observe the strong coupling between the spheres. The signal from the coupled modes is expected to be more pronounced when the incident light propagates parallel to the longitudinal axis of the PM. In order to control the alignment of the spheres constituting the PM we utilized a polystyrene substrate containing a three-dimensionally ordered array of pores of $\sim 5 \mu \mathrm{m}$ in size prepared through a thermocapillary convection. The surface layer of the substrate contains an array of open micro-wells of 3-5 $\mu \mathrm{m}$ depth. Only one pair of the 3 $\mu \mathrm{m}$ microspheres can be accommodated within each microwell, and the axis of the PM is close to the surface normal (Fig. 2, inset). 


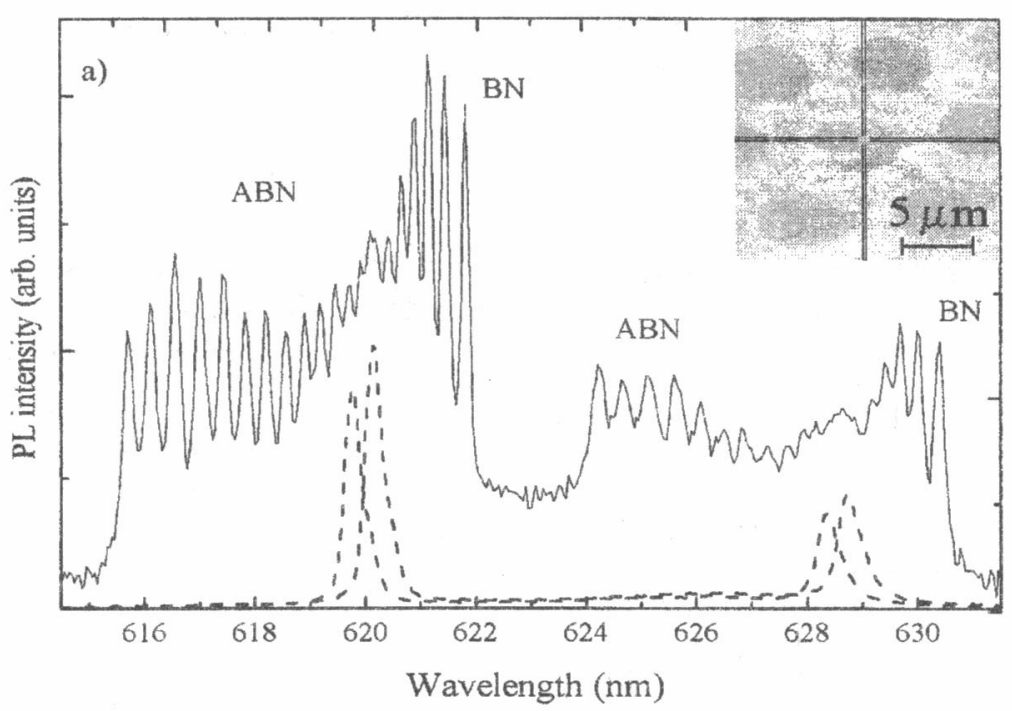

Fig. 2. - Comparison of PL spectra of a PM accommodated in a microwell (solid line) and PL spectra of noninteracting microspheres (dashed lines). Inset: microscope image of the PM in the microwell. The cross indicates the excitation position.

Figure 2 shows the PL spectrum of a PM accommodated in a microwell and the spectra of the individual microspheres prior to being manipulated into the microwell. The presented PL spectra clearly revealed major features unique to strong coherent coupling between the photonic states of the two microspheres forming the PM. One can clearly see a number of narrow peaks ( $m$-resonances) in the spectrum of PM on the short wavelength side of the TE and TM resonances, which are due to the presence of $m \neq \pm 1$ components. These peaks are grouped into the NB and ABN branches (Fig.2). The deconvolution of the lineshape of the $m$ resonances of the PM using Lorentian functions reveals the most remarkable experimental fact: the Q factor of $m$-resonances in spectra of the PM $(\sim 8000)$ (and therefore lifetime, $\tau$, of a photon in the resonant modes) exceeds the $Q$ value $(\sim 3000)$ and photon lifetime of individual spheres across the whole spectral region. This fact along with estimated value of BN/ABN splitting $(\sim 6-8 \mathrm{~nm})$ suggests the possibility for the development of new PM based photonic devices such as an optical delay line with controllable spectral and temporal tunability, which can be highly useful for a variety of applications in optical communication systems.

\section{REFERENCES}

1. K.J. Vahala, "Optical microcavities", Nature., vol. 424, p.839, 2003.

2. M. Artemyev and U. Woggon, "Quantum dots in photonic dots," Appl. Phys. Lett., vol.76, p. 1353.

3. Y.P. Rakovich, L. Yang, E.M. McCabe, J.F. Donegan, T. Perova, A. Moore, N. Gaponik, A. Rogach, "Whispering Gallery Mode Emission from a Composite System of CdTe Nanocrystals and a Spherical Microcavity," Sem. Sci. Tech., vol. 18, p. 914., 2003.

4. M. Bayer, T. Gutbrod, J.P. Reithmaier, A. Forchel, T.L. Reinecke, P.A. Knipp, A.A. Dremin, V.D. Kulakovskii, "Optical modes in photonic molecules," Phys. Rev. Lett., vol. 81, p. 2582, 1998.

5. K.A. Fuller, "Optical Resonances and Two-Sphere Systems", Appl. Opt., vol. 30, p. 4716, 1991.

6. H. Miyazaki, Y. Jimba, "Ab initio tight-binding description of morphology-dependent resonance in a bisphere", Phys. Rev. B, vol. 62, p. 7976, 2000. 